\title{
SEASONAL VARIATION AND RECENT STATUS OF TYPHOID FEVER IN A TERTIARY CARE HOSPITAL
}

\author{
Samreen Soomro $^{1,2}$, Samina Baig ${ }^{1}$, Saima Naseem ${ }^{1} \&$ Shaheen Sharafat ${ }^{l}$ \\ 1. Dow University of Health Sciences, Karachi. \\ 2. Dr. Panjwani center for molecular medicine and drug research, University of Karachi. \\ Corresponding Author Email: samreen@samerica.com
}

\begin{abstract}
In many developing countries, typhoid fever is endemic. Early diagnosis is important for appropriate treatment on time. Typhi dot is important diagnostic tool for early diagnosis of typhoid fever. In the current study from February, 2012 to May 2014, samples from 19433 febrile cases referred to Dow Diagnostic Research and Reference lab were screened for serum IgM and IgG for Salmonella typhi by Typhi dot test. Results showed that about 12501 patients had both IgM and IgG antibodies against Salmonella typhi. Male patients were more commonly affected than female patients. Highest frequency of positive cases was seen during the month of October. Age group >15-44 was most likely affected among different age groups. This study suggested that still we are far behind in improving sanitation and elimination of such controllable disease. Therefore prompt measures should be taken to control the disease especially during hot season by vaccination, improved sanitation and education as well.
\end{abstract}

\section{KEYWORDS}

Typoid fever, typhi dot, Salmonella typhi, immunochromatographic assay, anti- salmonella antibodies

\section{INTRODUCTION}

Typhoid fever is an emerging problem in developing countries. The causative agent of typhoid is Salmonella enteric, which is further categorized on the basis of serotype typhi. Typhoid is a major health issue in endemic areas, where the facilities of safe drinking water and poor sanitation.

It is estimated about 22 million new cases of typhoid occur each year and 200,000 of these resulting in death worldwide (Crump, 2004; Dewan, 2013). In Southeast Asia and Central South reported numbers of cases are more than 100 per 100,000 persons per year (Nagshetty, 2010). Pakistan is located highly endemic region and highest incidence rate 451.7 per 100,000 persons per year of typhoid fever, in comparison to the India incident rateis 214.2 per 100,000 persons per year (Dewan, 2013; Ochiai, 2008).

Major risk factors of disease transmission are contaminated food, drinking water, miserablesanitation condition, close interaction with typhoid patients or carriers, education, flooding, personal hygiene and travelling to endemic regions. In addition, climatic condition such as, rainfall (Black, 1985; Karkey, 2010; KellyHope, 2007; Wang, 2012).

The clinical picture of typhoid may not be explicated so early diagnosis of disease is important. Laboratory tests directly depend on the day of illness. A blood culture test based on 70-75\% of the first week of illness and is still as the gold standard for diagnosis (Hoffman, 1984). Rapid immunoassays have been commonly used for a few years with variable results in the world (Collantes, 1997; Ismail, 1991; Lu-Fong, 1999; Olsen, 2004). The present study aims to determine the outbreak of typhoid feverin our set-up and its seasonal variation

\section{METHODS}

The Study was performed in the Department of Microbiology, Dow Diagnostic Research and Reference Laboratory, Karachi, Pakistan, from February, 2012 to May, 2014. Total 19433 subjects were registered. The test is based on the presence of specific IgM and $\mathrm{IgG}$ antibodies against typhoid antigen, which is imprinted on nitrocellulose strips. The reaction tray was divided into 3 columns marked as $\mathrm{G}, \mathrm{M}$ and control .The $45 \mu \mathrm{L}$ of sample diluent was dispensed in the sample well and after that $45 \mu \mathrm{L}$ of buffer was added. The results were read and interpreted after 15 minutes.

\section{RESULT}

A total 19433 number of serum samples were tested for IgM and IgG antibodies against Salmonella typhi by means of Typhi dot test. Out of total registered cases, 10899 were male patients and 8530 were female population. Our results indicated about these, 6399 (32.9\%) male patients were positive and 6102 (31.4\%) female patients were positive for typhoid fever.(shown in figure1).

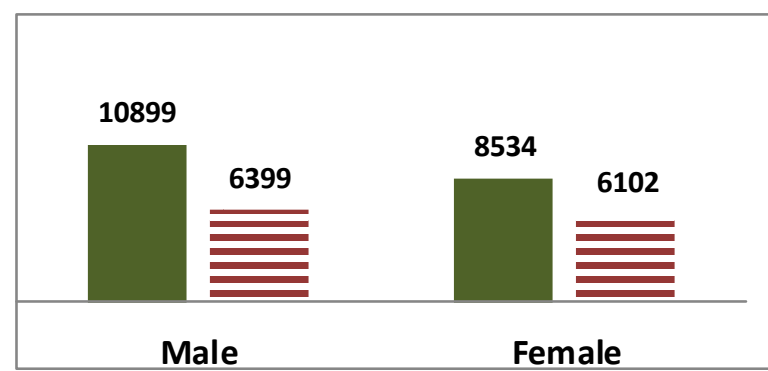

Figure 1: Typhi Dot Analysis of Febrile Cases: Total registered male and female population in this study shown as, while positive cases indicated as representing positive and registered cases during last 2.5 years. 
Out of the total positive cases i.e 12501, about 318(1.63\%) of patients show IgG antibodies alone and 4861 (25\%) showIgM antibodies alone. Additionally, 3652 (18.79\%) show both IgG and IgM antibodies. Positivity pattern of IgG and IgM among patients in different months was analyzed from February 2012 to May
2014. According to which higher frequency of positive cases was seen during the month of October and May. The table 1 shows the detailed frequency of positive cases along with type of antibodies found

positive.

\begin{tabular}{|c|c|c|c|c|}
\hline Months & Frequency & $\begin{array}{c}\text { IgG } \\
\text { positive }\end{array}$ & $\begin{array}{c}\text { IgM } \\
\text { positive }\end{array}$ & $\begin{array}{c}\text { Total } \\
\text { IgG+IgM }\end{array}$ \\
\hline Jan & 937 & 213 & 548 & 761 \\
\hline Feb. & 1483 & 337 & 701 & 1038 \\
\hline March & 2051 & 384 & 880 & $* 1264$ \\
\hline April & 1801 & 369 & 917 & $* 1286$ \\
\hline May & 2144 & 396 & 838 & $* 1234$ \\
\hline June & 1266 & 240 & 397 & 637 \\
\hline July & 1313 & 219 & 462 & 681 \\
\hline Aug & 1556 & 296 & 562 & 858 \\
\hline Sept & 2062 & 432 & 689 & 1121 \\
\hline Oct & 2615 & 584 & 1348 & $* * 1932$ \\
\hline Nov & 1483 & 337 & 749 & 1086 \\
\hline Dec & 722 & 169 & 436 & 605 \\
\hline
\end{tabular}

Table1: Typhoid Positivity Pattern Against Serum IgG and IgM: Results indicating mean positive cases against serum antibodies From February 2012 to May 2014.

In this study, when positive cases were analyzed according to different age groups it was found that the highest number of the patients belong to age group $>15-44$ years. However, $>44-50$ years were least affected. All these results are shown in figure 2 (a \& b).

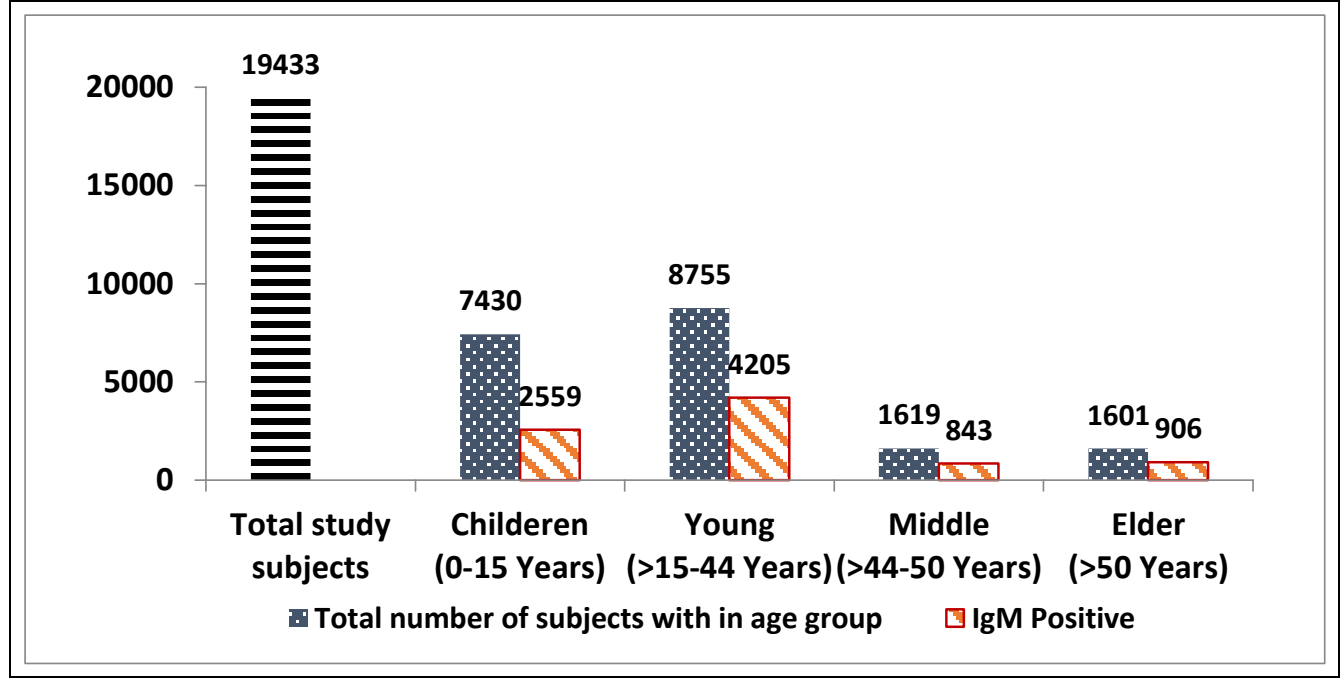

Figure 2a: Typhidot IgM Positive Cases Among Different Age-groups: shown as the total number of subjects and indicated the total subjects in different age group, while representspositive cases in each group. Results are cumulatively representing positive and registered cases during last 2.5 years. 


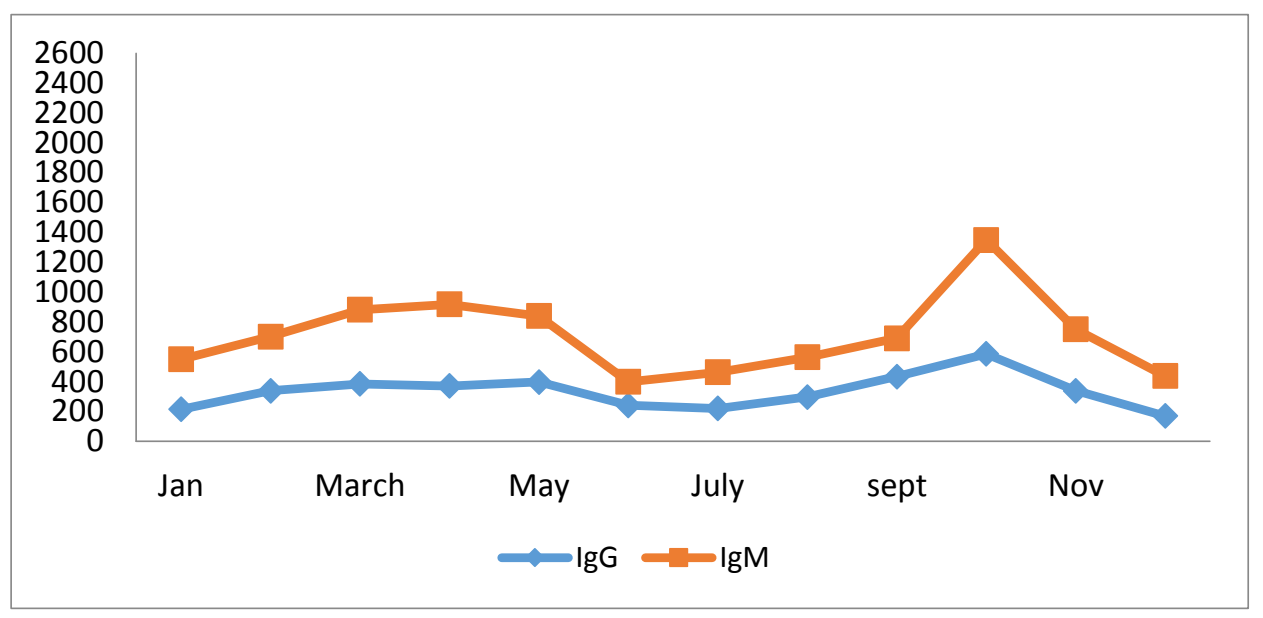

Figure 2b: Evaluation of Seasonal Variation among Typhoid Fever Cases: Results show mean number of typhoid positive case in each month for both antibodies IgG and IgM from February, 2012 to May, 2014.

\section{DISCUSSION}

The emergence of multidrug resistance among type strains is one of the major causes of difficulties faced during the treatment of typhoid fever.(Olsen, 2004) Delay in the diagnosis and initiation of effective treatment is associated with increased risk of complications during the course of the disease.(Krishna, 2011;) To meet the need of rapid and accurate diagnosis of typhoid fever, Typhi dot is the test of choice. It detects antibodies to outer membrane protein (OMP) antigen present on S. typhi (AbdAlrahman, 2014). Positive results can be obtained within 1hour and interpreted visually.[15]Although blood culture is considered as a gold standard (Jesudason, 2006) in the diagnosis of typhoid fever, but delay in its result has led to the use of other means of diagnostic tools. According to the different research study typhi dot was found to be $100 \%$ sensitive and $95.5 \%$ specific (Jesudason, 2002; Jesudason, 2006; Krishna, 2011).

In this study, out of 19433 patients, 8513 (43.9\%) have antibodies against Salmonella typhi. Male patients10899 (56\%) are more commonly affected than female $8530(44 \%)$. It is in favor of the results seen in a study done in Abbottabadin 2011 and Tamil Nadu in 2014 (Cinthujah, 2014; Hayat, 2011). Among the total positive cases, $4861(25 \%)$ have IgM antibodies (alone) and 318(1.63\%) of patients have $\mathrm{IgG}$ antibodies (alone) showing high rate of recent infection. When positivity pattern of antibodies during different months from 2012 to 2014 was analyzed, it was found that there is a peak incidence of positive cases during October whereas moderately high frequency was seen in March, April and May. It is consistent with the results of the study done by Olsen S. J, et al in Vietnam (endemic area for Typhoid fever) in 2004 (Olsen, 2004). Moreover, analysis of positive cases, according to the age group shows the majority of the patients belongs to the mean age of 37years and least affected age group was older age group. Mostly young people are affected which is in accordance with the results of study done at the Northern Institute of Medical Sciences (NIMS) and Ayub Teaching Hospital ( Abbottabad)in 2011where mean age of the study group was $26.31 \hat{\mathrm{A}} \pm 11.8$ (SD) years (Hayat, 2011).
Since, Typhidot is an inexpensive and reliable sero-diagnostic test, easily available commercially requiring no expert technical skill (Sherwal, 2004) Physicians can diagnose and initiate therapy early with the help of this test having high sensitivity and specificity (Hayat, 2011). In order to eradicate the disease at governmental level measures should be taken, to control the disease especially during hot season by vaccination, improved sanitation and education as well.

\section{CONCLUSION}

In brief, our study represents that typhoid is still a problem of developing countries like Pakistan and data was representing the prevalence of the disease in the month of October. Although most developed countries saw declining rates of typhoid fever throughout the first half of the 20th century due to vaccinations and advances in public sanitation, but still we need to improve sanitation and pollution of the waters and edibles in order to get rid of the disease.

\section{REFERENCES}

- Abd-Alhafeez, H., \& Nafi, M. (2014). Comparison of typhidot-eia and widal test in respect to polymerase chain reaction as diagnostic procedures for early diagnosis of typhoid fever. Journal of Biomedical and Pharmaceutical Research, 3(5), 18-20.

- Black, R. E., Cisneros, L., Levine, M. M., Banfi, A., Lobos, H., \& Rodriguez, H. (1985). Case-control study to identify risk factors for paediatric endemic typhoid fever in Santiago, Chile. Bulletin of the World Health Organization, 63(5), 899-904.

- Cinthujah, B., Amudha, V. P., Sucilathangam, G. (2014). Comparative Study of Widal and Dot Elisa in the Diagnosis of Typhoid Fever. Medical Science, 3(4), 303-304.

- Collantes, E., \& Velmonte, A. M. (1997). The validity of the typhidot test in diagnosing typhoid fever among Filipinos. Phil J Microbiol Infect Dis, 26(2), 61-63.

- Crump, J. A., Luby, S. P., \& Mintz, E. D. (2004). The global burden of typhoid fever. Bulletin of the World Health Organization, 82(5), 346-353 
- $\quad$ Dewan, A. M., Corner, R., Hashizume, M., \& Ongee, E. T. (2013). Typhoid Fever and its association with environmental factors in the Dhaka Metropolitan Area of Bangladesh: a spatial and time-series approach. PLoS Negl Trop Dis, 7(1), e1998.

- $\quad$ Hayat, A. S., Shah, S. I. A., \& Shaikh, N. A. I. L. A. (2011). Evaluation of Typhidot (IgM) in early and rapid diagnosis of typhoid fever. Professional Med J, 18(2), 259-264.

- Hoffman, S. L., Punjabi, N. H., Rockhill, R. C., Sutomo, A., Rivai, A. R., \& Pulungsih, S. P. (1984). Duodenal stringcapsule culture compared with bone-marrow, blood, and rectal-swab cultures for diagnosing typhoid and paratyphoid fever. Journal of Infectious Diseases, 149(2), 157-161.

- $\quad$ Ismail, A., Kader, Z. S., \& Kok-Hai, O. (1991). Dot enzyme immunosorbent assay for the serodiagnosis of typhoid fever. Southeast Asian J Trop Med Public Health, 22(4), 563-566.

- Jesudason, M., Esther, E., \& Mathai, E. (2002). Typhidot test to detect IgG \& IgM antibodies in typhoid fever. Indian J Med Res, 116, 70-72.

- Jesudason, M. V., \& Sivakumar, S. (2006). Prospective evaluation of a rapid diagnostic test Typhiot ${ }^{\circledR}$ for typhoid fever. Indian Journal of Medical Research,123(4), 513-516.

- $\quad$ Karkey, A., Arjyal, A., Anders, K. L., Boni, M. F., Dongol, S., Koirala, S., \& Baker, S. (2010). The burden and characteristics of enteric fever at a healthcare facility in a densely populated area of Kathmandu. PLoS One, 5(11), e13988.

- Kelly-Hope, L. A., Alonso, W. J., Thiem, V. D., Anh, D. D., Canh do, G., Lee, H., \& Miller, M. A. (2007). Geographical distribution and risk factors associated with enteric diseases in Vietnam. Am J Trop Med Hyg, 76(4), 706-712.

- $\quad$ Krishna, S., Desai, S., Anjana, V. K., \& Paranthaaman, R. G. (2011). Typhidot (IgM) as a reliable and rapid diagnostic test for typhoid fever. Annals of tropical medicine and public health, 4(1), 42-44.

- $\quad$ Lu-Fong, M., Ludan, A. C., Martinez, M. M., \& Raymundo, J. G. (1999). Dot EIA (Typhidot): an aid to the diagnosis of typhoid fever among Filipino children.Malaysian Journal of. Child Health, 8, 163.

- $\quad$ Nagshetty, K., Channappa, S. T., \& Gaddad, S. M. (2010). Antimicrobial susceptibility of Salmonella Typhi in India. J Infect Dev Ctries, 4(2), 70-73.

- Ochiai, R. L., Acosta, C. J., Danovaro-Holliday, M., Baiqing, D., Bhattacharya, S. K., Agtini, M. D., \& Clemens, J. D. (2008). A study of typhoid fever in five Asian countries: disease burden and implications for controls. Bulletin of the World Health Organization, 86(4), 260-268.

- $\quad$ Olsen, S. J., Pruckler, J., Bibb, W., Nguyen, T. M., Tran, M. T., Nguyen, T. M., \& Mintz, E. D. (2004). Evaluation of rapid diagnostic tests for typhoid fever. J Clin Microbiol, 42(5), 1885-1889.

- Sherwal, B., Dhamija, R., Randhawa, V., Jais, V., Kaintura, A., \& Kumar, M. (2004). A comparative study of Typhidot $\mathrm{M}$ and widal test in patients of Typhoid fever. Journal of Indian Academy of Clinical Medicine, 5(3).

- Wang, L. X., Li, X. J., Fang, L. Q., Wang, D. C., Cao, W. C., \& Kan, B. (2012). Association between the incidence of typhoid and paratyphoid fever and meteorological variables in Guizhou, China. Chinese Med J, 125(3), 455-460. 